\title{
Report of the Second Brazilian Symposium on Basic Research in HIV/AIDS
}

\author{
Angra dos Reis, RJ, Brazil \\ September 7-11, 1997

\section{Vera Bongertz, Mariza Gonçalves Morgado, Luiz Roberto Ribeiro Castello- Branco*, Bernardo Galvão-Castro**}

\author{
Laboratório de AIDS e Imunologia Molecular *Laboratório de Imunologia Clínica, Departamento de \\ Imunologia, Instituto Oswaldo Cruz, Av. Brasil 4365, 21045-900 Rio de Janeiro, RJ, Brasil **Laboratório \\ Avançado de Saúde Pública, Centro de Pesquisas Gonçalo Moniz-Fiocruz, Salvador, BA, Brasil
}

After the successful realization of the First Brazilian Symposium on Basic Research in HIV/AIDS in 1995, the Scientific Committe of the Symposium decided to continue holding such meetings, with two years intervals; so the Second Brazilian Symposium on Basic Research in HIV/AIDS was held in September 1997, in Angra dos Reis, Rio de Janeiro, Brazil.

As pointed out during the opening session by the president of the symposium, Dr Bernardo Galvão-Castro (Fiocruz, Salvador, BA), the success of the first symposium motivated an even greater interest in the second meeting. Remembering the efforts of Peggy and Hélio Pereira (honored by the symposium) in initiating AIDS research in Brazil and the fundamental importance of Lair Guerra de Macedo Rodrigues of the Brazilian Ministry of Health (honorary president of the meeting) in furthering public health efforts to control HIV-1 spread, Galvão-Castro closed his speech expressing our longing for the late "Betinho", the sociologist Herbert de Souza, who was the honorary president in the first symposium.

The director of the Oswaldo Cruz Institute, Dr José Rodrigues Coura, reminded us of the importance of AIDS and HIV infection in our tropical country, where co-infections with many pathogenic agents may occur differently from the subtropic/ temperate (Europe, USA) countries where the main efforts for development of chemotherapeutic agents and vaccine candidates are being carried out.

The work of the Brazilian Ministry of Health, through the National Coordination for Sexually Transmitted Diseases and AIDS (CN-DST/AIDS) was reported by Dr Cristina Pimenta at the opening session, showing us the shift in "risk groups" in AIDS in Brazil, with the increasing infection of women, and the necessity to control vertical HIV1 transmission. In a separate session, the ongoing work related to the National Laboratory Networks for immunological and virological load determi- nations of HIV-1 positive individuals, as well as for molecular epidemiology studies of HIV diversity in Brazil (Dr Cláudia Renata F Martins), to the medical assistance and antiretroviral and other drug distribution organized and supervised by the CN-DST/AIDS (Dr Valdiléa G Velloso), the epidemiological survey and monitoring of the epidemy (Dr M Rebecca O Gomes) and the close collaboration of the CN-DST/AIDS with Brazilian universities (Dr Luiza Paiva Silva) were presented as a round table.

\section{ANTI-HIV/AIDS VACCINES}

The review "Towards a global medical approach of AIDS: prospects for treatments and vaccines" was presented by Prof. Luc Montagnier (I Pasteur, Paris, France). Summarizing data of HIV variability and of the increasing number of reports on "mosaic" viruses, of anti-HIV immune response in acute infections and recent reports on co-receptors for HIV, he pointed out the necessity of a fast efficient control of HIV replication, wether by prophilactic or therapeutic vaccinations or chemotherapy.

Dr Jose Esparza (UNAIDS, Switzerland) reported on "UNAIDS HIV/AIDS Vaccine Strategy, Activity and Plans". After a brief review of the AIDS epidemic in worldwide terms, he summarized the main problems still to be faced in the development of an effective anti-HIV/AIDS vaccine. The importance of developing vaccines potentially efficient not only against the B subtype of HIV-1, prevalent in Europe and North America, but also against genotypes $\mathrm{C}$ and $\mathrm{A}$, much more prevalent in world terms ( 36 and $23 \%$ vs $16 \%$ ); the increasingly dynamic prevalence of HIV subtypes, the need to understand the importance of HIV genotypes in terms of viral control; the importance of establishing correlations between anti-HIV immune response and protection; the urgent need to identify the factors responsible for individual anti- 
HIV "resistance" such as co-receptor interaction, Dr Esparza concluded with an overview of recent results obtained in different vaccine trials. Studies carrying out "SHIV" (chimera of SIV \& HIV) organisms indicate a direct correlation between virulence of the infecting agent and level of protection acchieved; nef-compromised agents, "naked" DNA and others. Up to now 25 phase I/II trials have been carried out worldwide. Phase III trials have not been started yet, but shortly a canary pox vector expressing HIV-1 env/gag/pol will be tested under supervision of UNAIDS in Kampala, Uganda (chairman: B Bloom). He pointed out that, if trials are started today, earliest results will be obained between the years of 2003 and 2007. It has to be remembered that the increasing use of triple therapy will affect incidence of HIV-1 and therefore cost and period of vaccine trials.

Studies of new anti-HIV drugs, protease inhibitors and anti-HIV-1 reverse transcriptase, were presented by groups of the Universidade Federal do Rio de Janeiro and of the Universidade Federal Fluminense.

\section{IMMUNOPATHOLOGY OF HIV INFECTION}

Dr Giuseppe Pantaleo (Centre Hospitalier Universitaire Vaudois, Lausanne, Switzerland) presented data of studies analysing $\mathrm{T}$ cell clones from HIV-1 infected individuals. The study of Tcell receptor (TCR) Vb chains in individuals with "progressive disease" showed a statistically significant greater loss (or sharp decrease, below detection limits) of TCR Vb clones in "progressors" in comparison to "non-progressors" $(\mathrm{p}<0.0001)$. A positive correlation between polyclonality of the TCR Vb and control of disease progression could be established. Comparison of TCR Vb clones indicated a greater concentration of CD8-T cells in peripheral blood than in lymph nodes during primary HIV infection which, together with the specific antibody response, may be responsible for the sequestration of the virus in the lymph nodes.

Dr Robert Bollinger (Johns Hopkins University, Baltimore, USA) briefly reviewed data on cytotoxic T lymphocyte (CTL) activity in HIV infection. The importance of a polyclonal CTL response in HIV-1 infection and anti-HIV/AIDS immunization has been demonstrated by studies from his group, as well as the effectiveness of a cross-cytotoxic immune response. No correlation between genotype and "CTL-type" has been established yet, but cross-genotype CTL responses have been detected. After briefly explaining the methodology used, based on using labelled autologous B-cell lines infected with vaccinia virus carrying genes for different HIV-1 proteins as target cells, he reported that his group has developed more than $40 \mathrm{HIV}-1 \mathrm{C}$ genotype epitopes expressed in vaccinia vectors and have identified at least one epitope identic for HIV-1 C and B subtypes. Comparative analyses of autologous and heterologous CTL epitopes, using vaccinia vectors produced to express the epitope present in one infected individual's virus, indicated that altough cross-reactivity could be shown to exist, autologous cytotoxic activity was more efficient than heterologous activity. For use in protective measures, CTL will necessarily have to be cross-effective, associated to multi-cultural MHC antigens and, probably, active at mucosal sites.

Dr Otoniel Martinez Maza (UCLA, Los Angeles, CA, USA) presented results of B lymphocyte hyperactivation early in HIV infection, with details on activation markers. Increased IL-6 (BSF2) secretion, and elevated levels of IL-6 receptor (CD126), increases in B7.2, CD95 "Fas" and CD40 are observed in HIV infection, wether due to EBV co-infection, direct or indirect superantigen effects, chronical antigenic stimulation or B lymphocyte stimulation through cytokines. Increased IL-6 secretion was shown to be induced in vitro by the HIV gp41 antigen. Lymphomas are 60-200 times more frequent in HIV infection than in the general population, and a positive correlation with EBV infection $(40 \%)$ and c-myc gene translocation (79\%) has been reported. Presence of lymphomas in HIV infection was associated to soluble CD23 (74\%) and soluble CD27 (100\%). Detection of high levels of sCD2, sCD27, IgE and IL-6 in HIV infection could be correlated to development of lymphomas one to three years later (see detailed report in this issue).

Dr Andrew Freedman (University of Cardiff, Wales, UK) and his group studied hematopoietic suppression in HIV infection. The development of an in vitro culture system for bone marrow cells permitted the identification of the HIV infected cell types: only monocytic cells were found to be infected. Treatment of infected cultures with AZT or ddI showed a greater influence of AZT on cell viability and function. The group was also successful in establishing thymocyte cultures, using IL12 and Flt-3 (fetal hepatic kinase) complements. The presence of CD34+CD2- precursor cells was shown to be necessary for HIV infection of thymus cells.

Dr Anne Hosmalin (Hôpital Pitié-Salpetriére, Paris, France) presented her results of a study of splenic dendritic cells in HIV infected individuals. Her isolation procedures permitted HIV detection by PCR with a sensitivity of $1 / 100,000$ cells, and allowed detection of HIV infection in different spleen cells: CD4+ T cells (1/60), dendritic cells $(1 / 3,000)$, mononuclear splenic cells $(1 / 1,500)$ and 
adherent splenic cells $(1 / 2,100)$. Intermediate values of HIV infection 10 to 14 times higher than detected in monocyte/macrophage cells were determined for dendritic cells. A characteristic high expression of CD83 was found in dendritic cells from HIV infected individuals.

Upon invitation, Dr Hosmalin also presented data from Patrice Debré and Brigitte Autran, on expansion of the CD4+CD7- cell subset in HIV infection and reduction to normal levels after triple chemotherapy, parallel to the increase in total CD4+ cells (see detailed report in this issue).

Dr Michael Hendry (California Dept. Health Services, Berkeley, CA, USA) presented results of serotyping analyses. Good identification of B, B", $\mathrm{C}$ and A genotype HIV-1 infection by serological analyses was shown, with correct distinction of $\mathrm{B}$, $\mathrm{C}$ or E HIV-1 genotype infections. Correlation to neutralization susceptibility or viral load was not detected. A study of vertical HIV-1 transmission in Zimbabwe permitted identification of a much higher proportion of in utero HIV-1 transmission $(77 \%)$ than reported for USA (25-33\%), leading to a hypothetical correlation of $\mathrm{C}$ genotype infection and in utero HIV transmission.

Dr Fernanda Martinez Salazar (Instituto Nacional de Diagnóstico y Referencia Epidemiologica, Mexico City, Mexico) presented results of a study analyzing the lymphoproliferative response (LPR) of Mexican children, offspring of HIV-1 infected mothers, against a panel of synthetic peptides. Two of $11 \mathrm{HIV}-1$ infected children and 5 of 13 non-infected children showed LPR against the peptides chosen, indicating the possibility of a "protection" in uninfected children.

Several reports of immunopathological studies carried out in Brazil were presented. Dr LR Castello-Branco (Fiocruz, Rio de Janeiro, RJ) presented data from an oral immunization trial of HIV-1 infected individuals with cholera vaccine: a positive correlation between numbers of circulating CD4 lymphocytes and the ability to secrete anti-cholera Toxin B (CTB) antibodies (IgG detected in 4/12 individuals) was established, and a slight transient elevation of viral load occurring shortly after immunization was observed in all volunteers but there was no deterioration in clinical outcome (see detailed report in this issue). Dr Alberto Duarte (Universidade de São Paulo, São Paulo, SP) presented a summary of cytokine studies carried out by his group indicating that, in general, HIV-1 infected individuals had elevated concentrations of IL-10 in blood, as well as slightly higher IFNg levels than non-infected individuals. IL-4 concentrations are quite low, even after phytohemagglutinin stimulation, and a positive corre- lation between disease progression (indicated by lower numbers of circulating CD4 lymphocytes) and high levels of IL-10 and IFNg could be noted (see detailed report in this issue). A review of results on humoral anti-HIV-1 immune response in Rio de Janeiro was presented by Dr Vera Bongertz (Fiocruz, Rio de Janeiro, RJ). Seroreactivity studies with V3 peptides indicated a high cross-reactivity between $\mathrm{B}$ and $\mathrm{F}$ subtype plasma, but statistically significant lower cross-reactivity $(\mathrm{p}<0.0001)$ with the Brazilian GWG variant of the B subtype (known as B"). This lack of crossed recognition of V3 peptides was not reflected in crossed neutralization assays, where no significant difference of neutralization of $\mathrm{B}, \mathrm{B}$ ” or $\mathrm{F}$ primary isolates by heterologous B, B" or F plasma could be observed (see detailed report in this issue).

Several works submitted as posters were discussed in an Immunopathology session, with recent data found in studies of Cytokines and Interleucins in HIV infection, immune response (humoral and lymphoproliferative) against synthetic HIV peptides, among others.

\section{CO-INFECTIONS}

Reports on co-infections common in Brazil, such as tuberculosis/HIV and HTLV/HIV were presented. Dr Carlos R Brites Alves (Universidade Federal da Bahia, Salvador, BA) analyzed the viral load of HIV-1 infected individuals co-infected with HTLV, which was shown to be higher than the one observed in non-co-infected individuals within the same group of patients. Dr Afrânio Kritsky (Universidade Federal do Rio de Janeiro, Rio de Janeiro, RJ) presented a review of HIV and Mycobacterium tuberculosis in Brazil in comparison to data reported outside of Brazil (see detailed report in this issue). Dr M Glória Bonecini Almeida (Fiocruz, Rio de Janeiro, RJ) presented results on a study of bronchoalveolar and peripheral mononuclear cell iNOS detection and cytokine production. Bronchoalveolar cells expressed less iNOS when obtained from HIV/M. tuberculosis co-infected individuals than from non-HIV infected tuberculosis patients, changing the clinical outcome of both infections (see detailed report in this issue). Dr Ricardo Ishak (Universidade Federal do Pará, Belém, PA) reported data on HTLV/HIV coinfection in Pará, with a $8 \%$ prevalence of co-infection, higher for HTLV-II than HTLV-I (see detaied report in this issue). Dr Regina Keller (Universidade Federal do Espírito Santo, Vitória, ES) reported detection of HHV8 (Human Herpes Virus 8) in six of eight AIDS patients with Kaposi's sarcoma.

The development of research in the association 
of Tropical Diseases and HIV was demonstrated by the high number of abstracts presented by groups from different regions of our country. Association between HIV and M. tuberculosis, HTLV, Blastocystis hominis, Chlamidia pneumoniae and C. trachomatis, Leishmania braziliensis, Trypanosoma cruzi were described, and the majority of the results were preliminar but have shown the importance of the first symposium, when the importance of co-infections in our country was specially stressed.

\section{HIV POLYMORPHISM}

Several authors presented data on studies of HIV polymorphism in Brazil. Dr Bernardo GalvãoCastro presented data from the National Network for HIV Isolation and Characterization, with results referent to 22 samples from Rio de Janeiro, 8 from São Paulo and 5 from Belo Horizonte, including data on isolation, phenotyping, genotyping, sequencing, seroreactivity and neutralization of the primary isolates. Dr Mariza Morgado (Fiocruz, Rio de Janeiro, RJ) presented data on the first subtype D HIV-1 identified in Rio de Janeiro, including homology analyses of $\mathrm{C} 2 \mathrm{~V} 3$ sequences with Southafrican D subtype. Moreover, her results from a genotype study of Rio de Janeiro $(n=131)$ indicated an absence of correlation between exposure group and HIV genotype in this sample (see detailed report in this issue). Dr Ester Sabino (Fundação Pró-Sangue, São Paulo, SP) presented results of genotype analyses of patients identified in blood donors from São Paulo capital and from Santos, SP, with a distinguishing prevalence of the F subtype in São Paulo and of the C genotype in Santos. Her results confirmed that no relationship between exposure and genotype or viral load and genotype could be established. Seroreactivity assays indicated a good relationship between $\mathrm{C}$ geno- and serotype. Dr Ana Carolina P Vicente (Fiocruz, Rio de Janeiro, RJ) presented results from a genotype analysis carried out on env and pol (protease) genes of HIV-1 samples from Manaus, AM, leading to the identification of a great number (4/ 9) of mosaic HIV-1 (B/F) genomes in the population studied. Dr Amilcar Tanuri (Universidade Federal do Rio de Janeiro, Rio de Janeiro, RJ) analyzed the HIV-1 protease gene of 70 samples from Rio de Janeiro, identifying a greater polymorphism in F subtype than detected in B subtype, which presented similar polymorphism of the protease gene as described for North-American HIV-1 B subtype.

Several studies submitted as posters were discussed in a Molecular Biology and Biochemistry session, and presented data on HIV-1 polymorphism in different geographical regions of Brazil, in different HIV-1 transmission groups, detection of recombinant samples and a study of protease gene polymorphism. Results of polymorphism studies carried out in Argentina, as well as results obtained analysing Bolivian samples, were presented.

\section{VIRAL LOAD}

Dr Giuseppe Pantaleo (Centre Hospitalier Universitaire Vaudois, Lausanne, Switzerland) presented data on viral load analyses carried out by his group. In reference to lymph node HIV load, statistically significant $(p<0.01)$ greater viral loads in individuals with more advanced disease could be detected. This greater viral load has been shown to reflect a greater load per cell and not a greater number of infected cells. A conclusion of his work is the possibility that plasmatic HIV load determined in the first months of infection may not reflect the total viral infection of the patient, while measurements after the 7th month of infection show a good correlation with disease progression and could therefore be used in the prognosis of disease progression. The data reinforce the necessity of chemotherapy as early as possible after infection for greater efficacy.

Dr Fabian Fay (Centro de Diagnostico Molecular, Rosário, Argentina) compared NASBA and bDNA quantitative viral load assays in their sensitity for measuring HIV-1 B or F subtype, concluding that the plasmatic load of B subtype HIV-1 infected patients was better detected using NASBA.

Studies carried out analyzing viral load in Brazilian patients were also presented. Dr Lucia Aleixo (Universidade Federal de Minas Gerais, Belo Horizonte, MG) presented data of an AZT trial in HIV-1 infected pregnant women in Brazil, reporting similar results to the ATCG 076 trial: a transmission percentage of 6.3 for treated vs $31 \%$ for non-treated women. A strong correlation between viral load and vertical HIV-1 transmission was noted, although exceptions were detected. A possibility that AZT, besides reducing viral load, may affect viral infectivity was discussed to explain absence of vertical transmission with high viral load in the mother's plasma. Dr João Renato Rebello Pinho (Instituto Adolfo Lutz, São Paulo, SP) compared viral load measurements with other markers of disease progression, finding good correlation only with b2-microglobulin levels. Although not correlating to viral load, the number of CD4 lymphocytes correlated with p24 antigen detection $(\mathrm{p}<0.001)$. The two viral load assays used showed a good correlation (79.3\%), although the bDNA assay showed a higher sensitivity than 
NASBA (see detailed report in this issue). Dr Ricardo Diaz (Universidade Federal de São Paulo, São Paulo, SP) reviewed data on disease progression, with increasing viral loads, HIV-1 diversity and proportion of syncytium-inducing variants.

The Symposium's Scientific Committee indicated Ivan Neves Junior, Laboratory of AIDS and Molecular Immunology, Department of Immunology, Instituto Oswaldo Cruz, Fiocruz, Rio de Janeiro, to receive the Peggy \& Hélio Pereira prize, for presenting his results on "Cellular Immune response in HIV-positive household contact from HIV-infected or not tuberculosis patients" (Abstract 32).

An abstract book was printed with summaries of all posters and is available at The Fiocruz Library (information available at the Internet: http:/ /www.fiocruz.br or by e-mai:l bibmang@dcc001. cict.br).
The presidency of the Brazilian Symposium on Basic Research in HIV/AIDS was transferred from Dr Bernardo Galvão-Castro (Fiocruz, Salvador, BA) to Dr Alberto Duarte (Faculdade de Medicina, Universidade de São Paulo) and the Third Brazilian Symposium on Basic Research in HIV/AIDS will be held in São Paulo, in 1999.

The symposium was sponsored by Fiocruz and Brazilian Ministry of Health - (CN-DST/AIDS), FAPERJ, FINEP, CNPq, CAPES and Embratel, with participation of Furnas, Lablaser, Organon Teknika, Akzo Nobel, Xerox, PE Applied Biosystems, CerMed, and the help of Multimeios/ CICT/Fiocruz.

This is a personal view of the Second Brazilian Symposium on Basic Research in HIV-1/AIDS, with special emphasis on results presented but not published in this journal, as interpreted by the authors. 
372 Meeting Report - Vera Bongertz et al. 